\title{
The Performance and Radiation Hardness of the Outer Tracker Detector for $\mathrm{LHCb}$
}

\section{Evelina Gersabeck}

Ruprecht-Karls-Universität Heidelberg on behalf of the LHCb OT collaboration 


\section{THE LHCB DETECTOR}

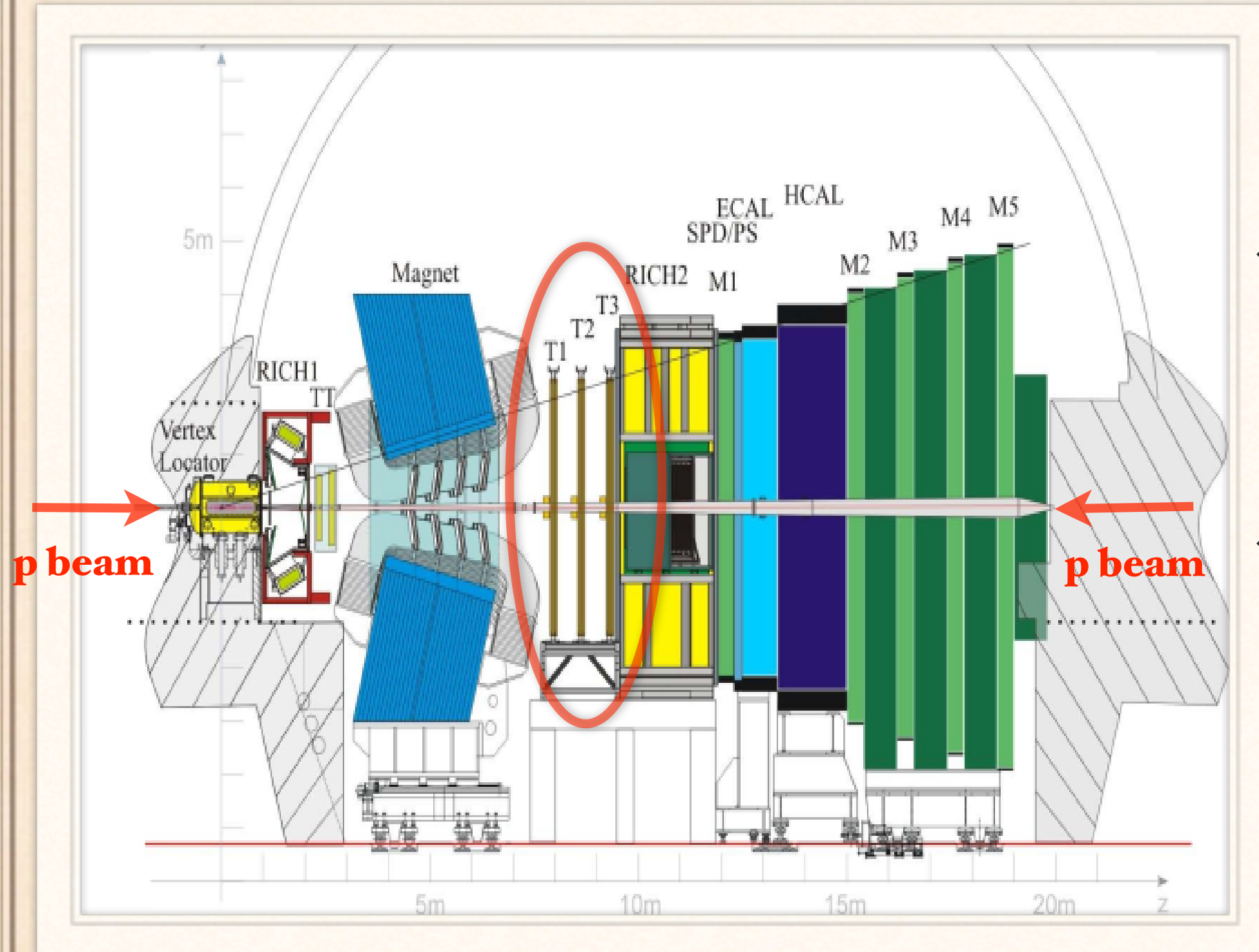

* Single arm spectrometer designed for heavy flavour physics measurements

Consists of Vertex Locator (VeLo), $2 \mathrm{RICH}$ detectors, silicon trackers, dipole magnet, outer tracker, ECAL, HCAL, muon system 


\section{OUTER TRACKER}

* Gaseous straw tube technology detector

4. 3 stations each with 4 layers of modules in $\mathrm{X}-\mathrm{U}\left(+5^{\circ}\right)-\mathrm{V}\left(-5^{\circ}\right)-\mathrm{X}$ geometry

* One detector layer is built of 14 long and 8 shorter modules

- Covers area of approximately $5 \times 6 \mathrm{~m}^{2}$

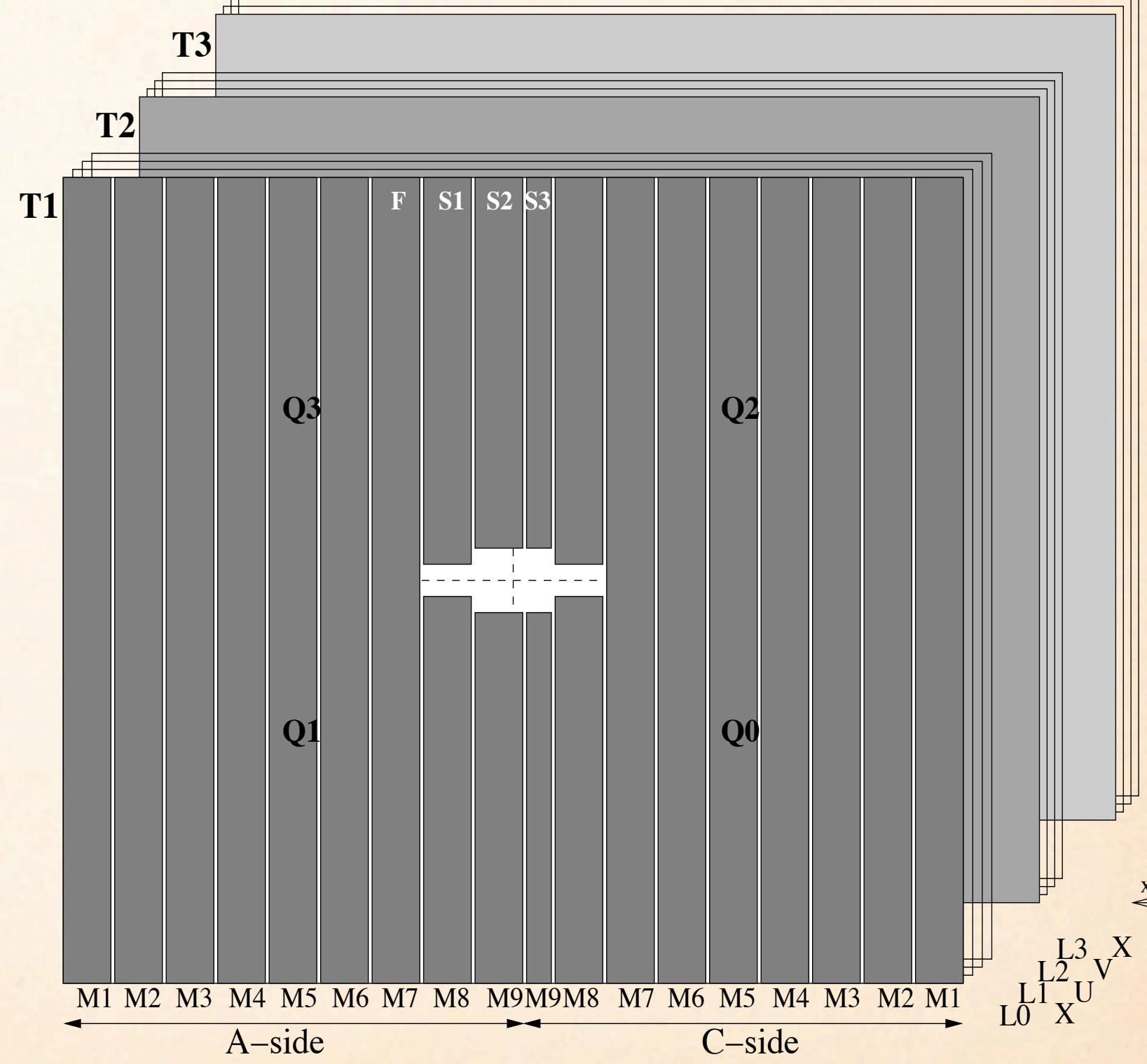




\section{OT STATIONS}

Each station is split into two halves, retractable on both sides of the beam line (on C-frames)

Number of modules: 216

Long module length: $5 \mathrm{~m}$

Module width: $340 \mathrm{~mm}$

. Inner region around the beam pipe: instrumented with Si strip detectors

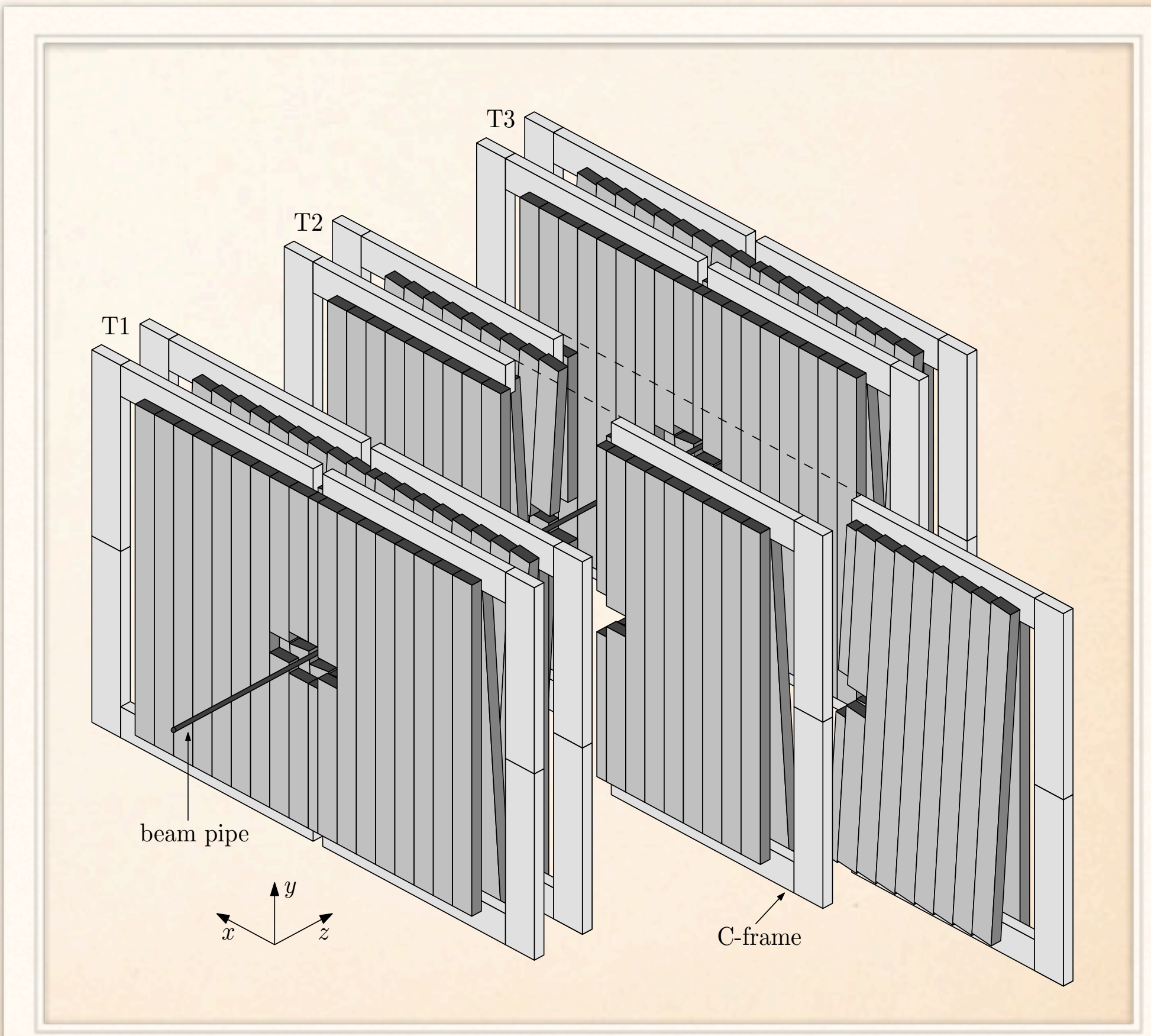




\section{OT MODULES}

Each module consist of 2 staggered straw tube monolayers

Numbers of straws in a monolayer: 64

. Total number of straws: 53760

- Inner diameter of straw tubes: 4.9 $\mathrm{mm}$

Straw tube length: 2.4 m

. Glue: Araldite AY103-1

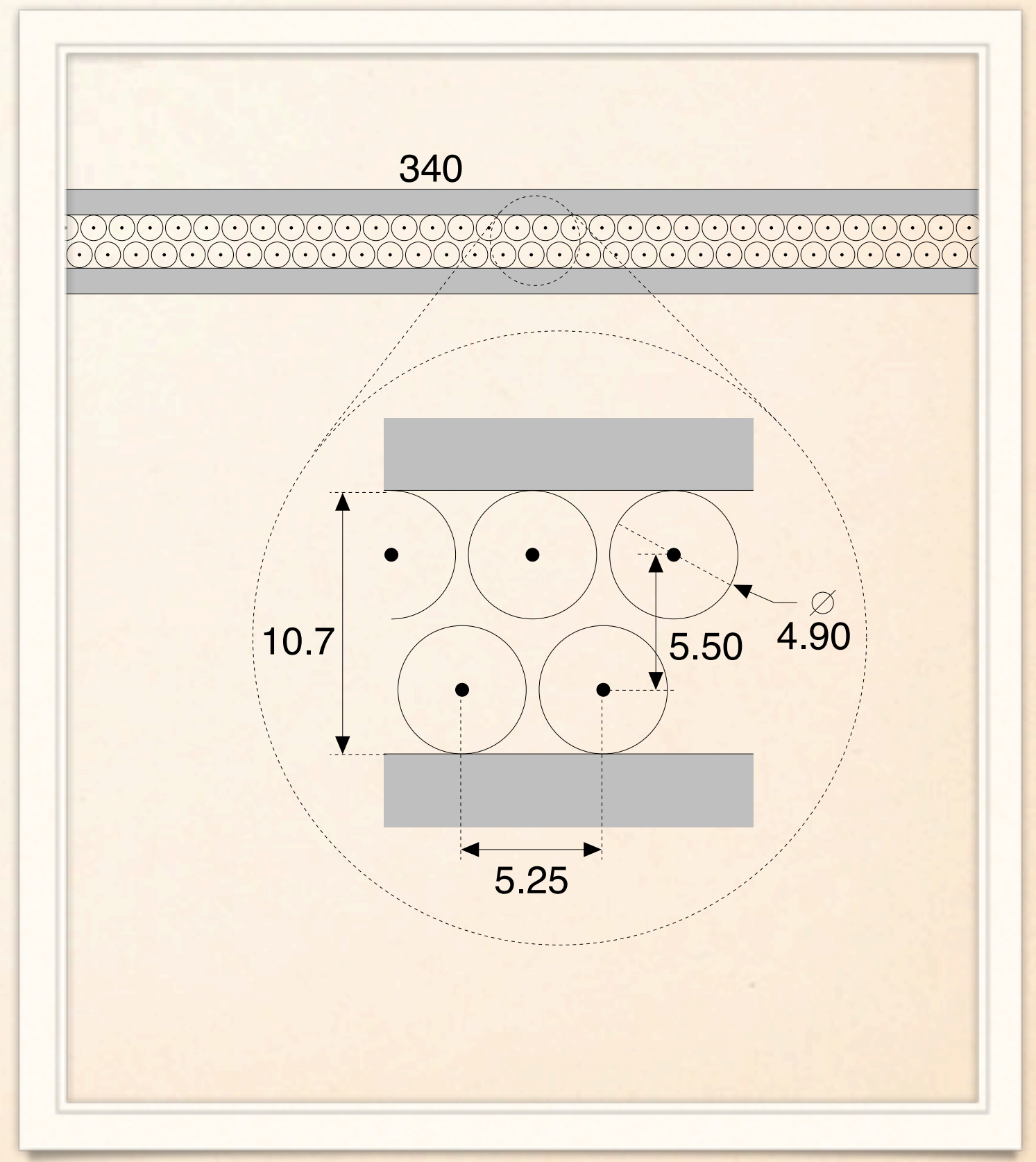




\section{OT STRAWS}

- Straw tube material: Kapton XC / Kapton / Aluminium

Anode wire diameter: $25 \mu \mathrm{m}$

(. Material of the anode: Gold plated Tungsten

Anode at $+1550 \mathrm{~V}$

Gain: $5 \times 10^{4}$

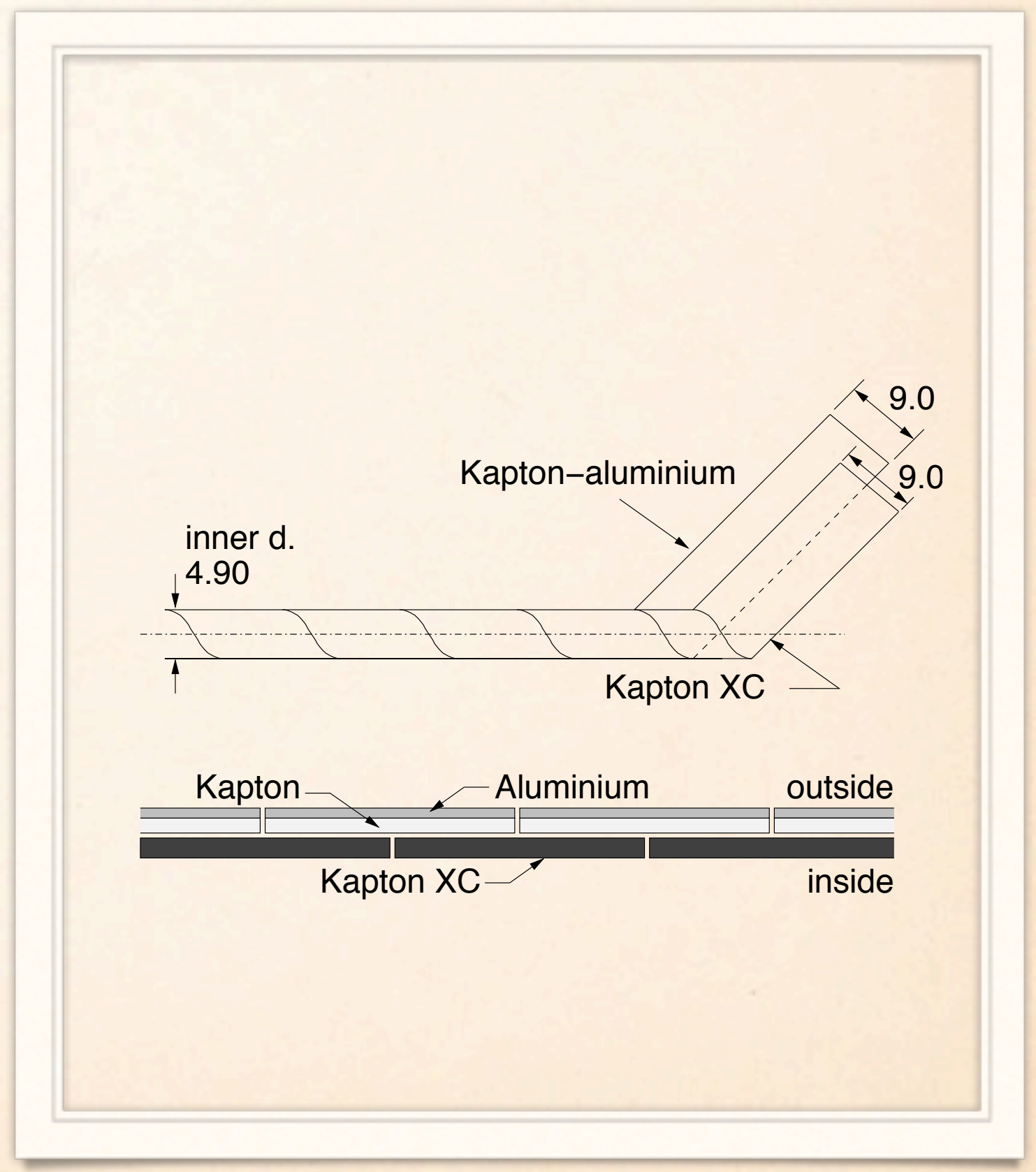




\section{TR-RELATION}

. The position of the hits in the OT is determined by measuring the drift-time to the wire of the ionisation clusters

The drift-time -distance relation (TR-relation) is used to convert the drift time to particle position.

$$
t_{\text {drift }}(r)=20.5 \mathrm{~ns} \cdot \frac{|r|}{R}+14.85 \mathrm{~ns} \cdot \frac{r^{2}}{R^{2}}
$$

The TR-relation is calibrated on data by fitting the distribution of drift-time as a function of the reconstructed distance of closest approach between the track and the wire

First iteration: TR-relation obtained from beam tests

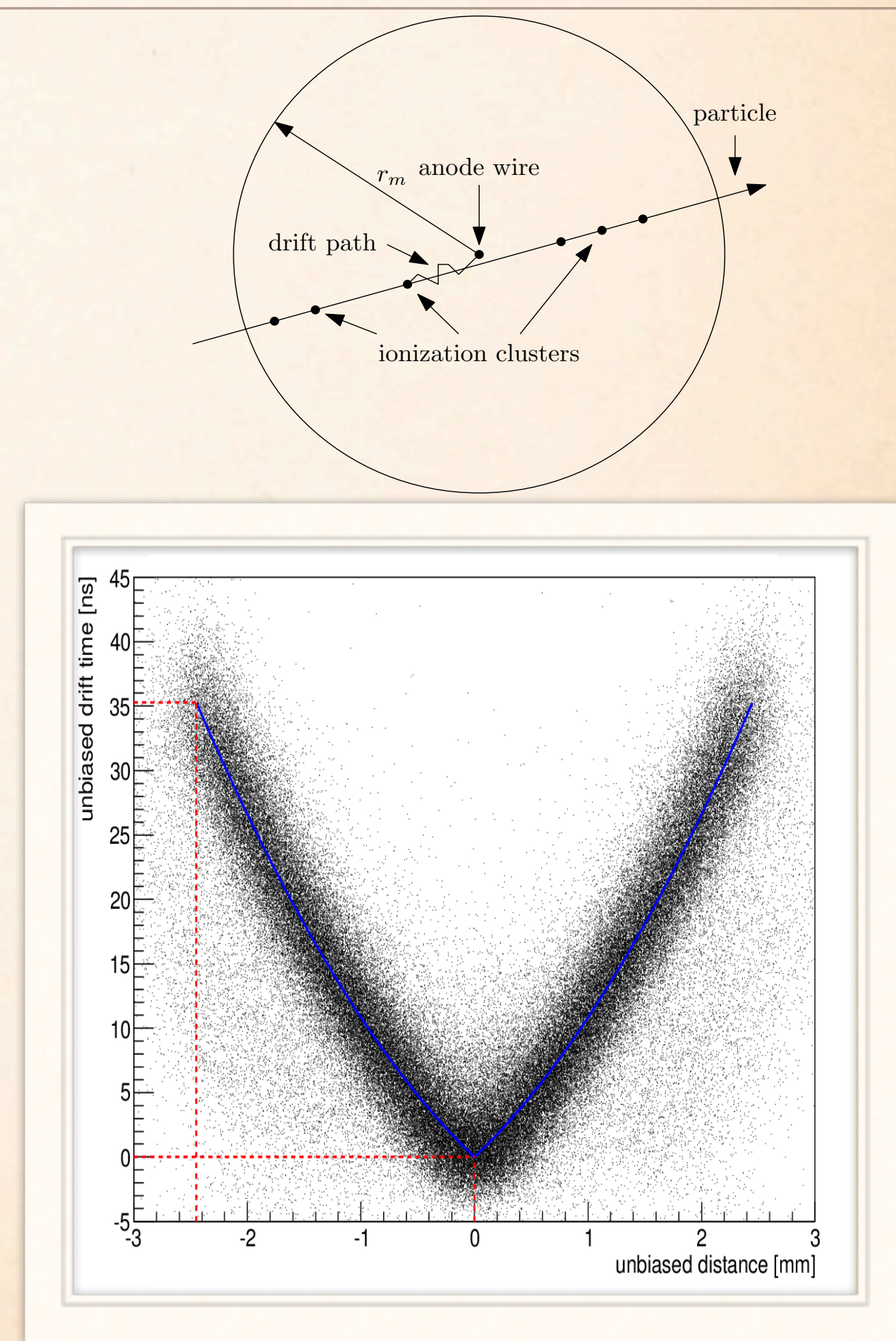




\section{OT ELECTRONICS}

Modular design of the FE electronics

* The main components:

-\& the high voltage (HV) board

-8 the ASDBLR amplifier chip: the complete analog signal processing (amplification, shaping, baseline restoration, and discrimination)

\& the OTIS drift time digitization chip

-8 the GOL auxiliary (GOL/AUX) board: the power connection, the interface to the fast control (beam crossing clock BX, triggers, resets) and the interface to the slow-control

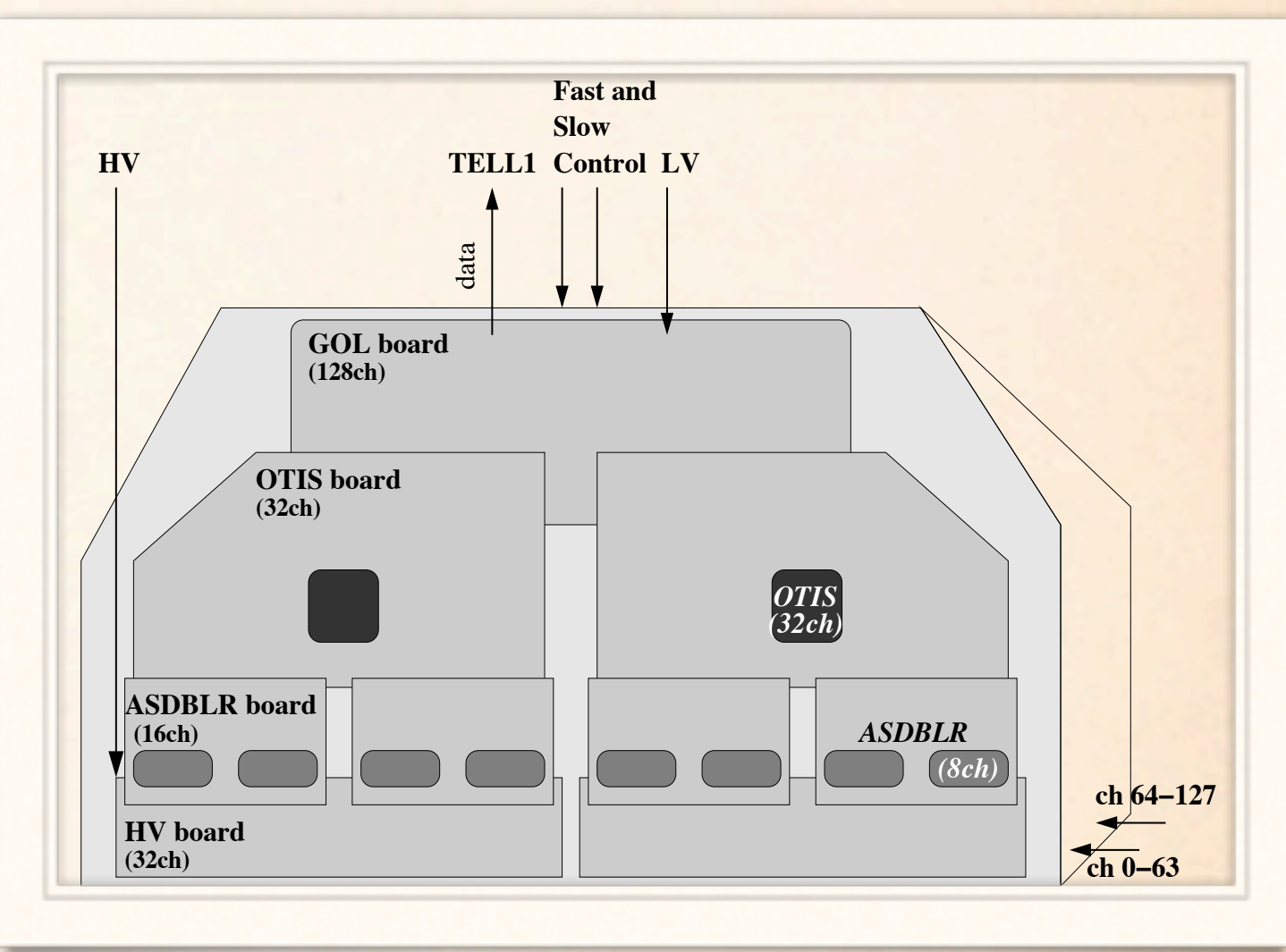

The drift times are digitized for each $25 \mathrm{~ns}$ (the LHC design value for the minimum bunch crossing interval) and stored in a digital pipeline $(4 \mu \mathrm{s})$ to await the lowest-level trigger (Lo) decision.

On a positive Lo decision, the digitized data in a window of $75 \mathrm{~ns}$ is transmitted via optical links (I.6 GBit/s) to TELLi boards in the LHCb DAQ system 


\section{THRESHOLD AND DELAY SCANS}

Possibility to inject a test pulse at the ASDBLR test input

2 kinds of test runs:

Test pulse at varying threshold settings: check the threshold uniformity and cross-talk

Test-pulse (at fixed threshold) at increasing test-pulse delay settings: determine the timelinearity

Relative variation is well within expected values
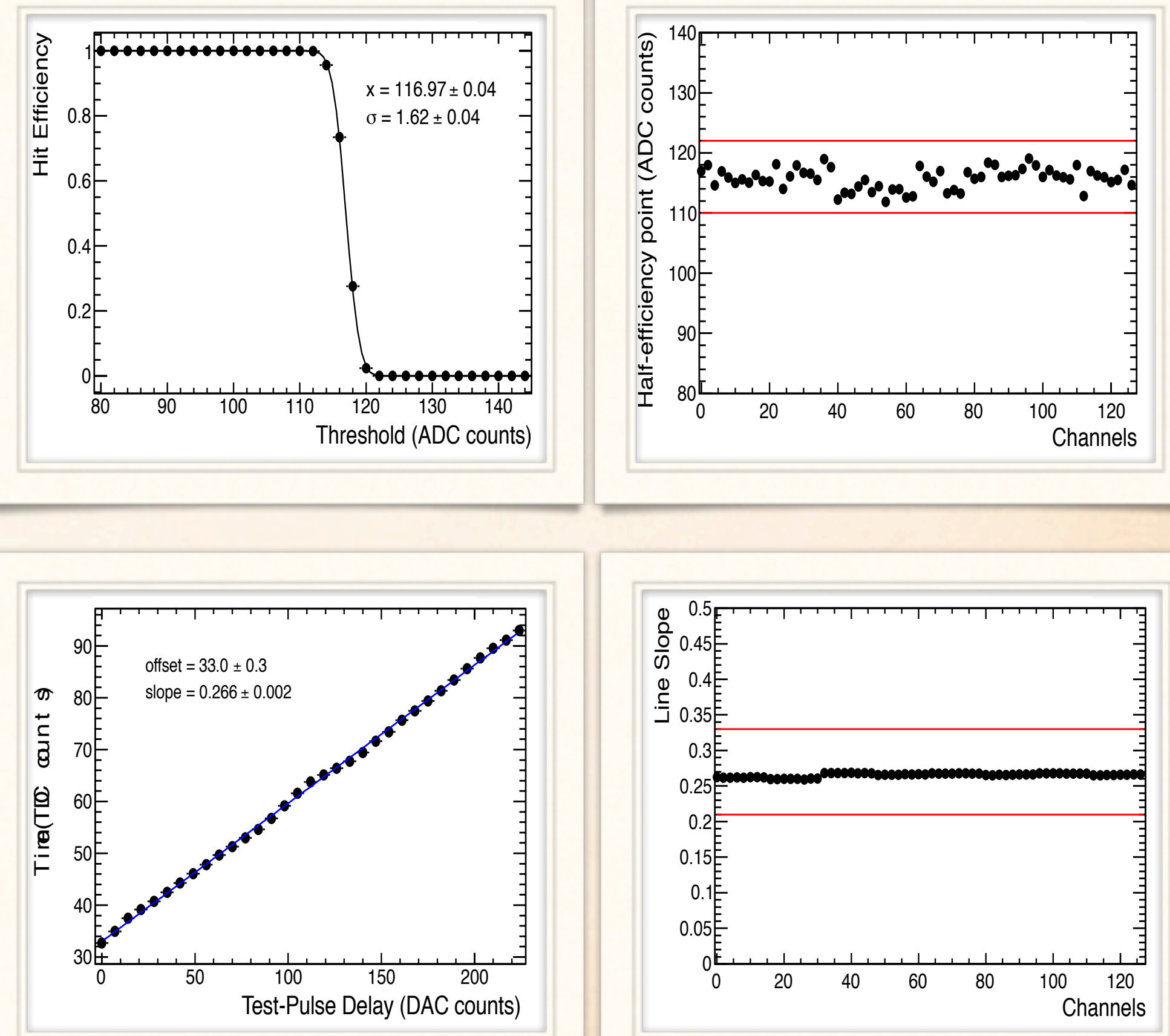


\section{NOISE}

* Noise due to: dark pulses from the detector, bad FE -electronics shielding, or bad grounding

(A) Check occupancy at increasing values of the amplifier threshold (nominal value $800 \mathrm{mV}$ ):

•E Using random triggers

.8. About $2 \%$ of the FE boxes exhibited noise pattern

-8. At the nominal threshold of $800 \mathrm{mV}$ only $0.2 \%$ of the channels exhibited a noise occupancy larger than $0.1 \%$ (usual occupancy $\sim 10^{-4}$ )
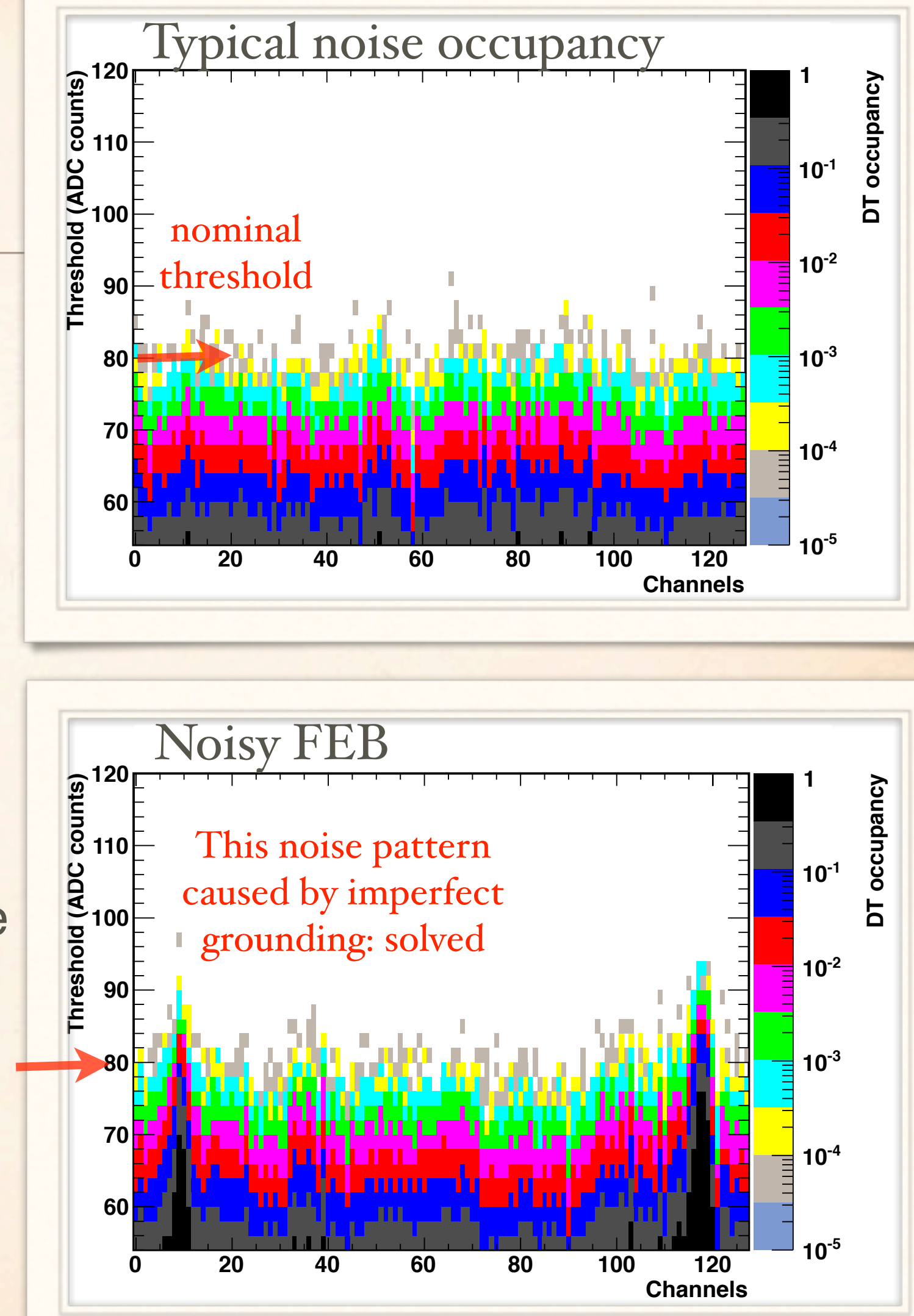

* Completely negligible for data taking 


\section{GAS SYSTEM}

Ar / $\mathrm{CO} 2 / \mathrm{O}_{2}(70 / 28.5 / 1.5)$ : fast drift time below 50 ns

Ô. OT continuously flushed: no recycling of gas

佥 Gas quality constantly analysed and monitored

Level of impurities $\left(\mathrm{H}_{2} \mathrm{O}\right)$ monitored: low and stable

Pre-mixed bottles containing in total about $100 \mathrm{~m}^{3}$ of $\mathrm{Ar} / \mathrm{CO}_{2} / \mathrm{O}_{2}$, is automatically activated in case of electrical power failures of the main gas system, or during various interventions

Back-up system is used regularly, and is well working 


\section{GAS GAIN TRENDS}

* Gas gain is monitored with two custom built OT modules, of $1 \mathrm{~m}$ length, which are irradiated by a ${ }^{55} \mathrm{Fe}$ source $\left(\mathrm{K}-\mathrm{a}{ }^{55} \mathrm{Mn}\right.$ line used as calibration reference)

Take pulse height spectrum (every $15 \mathrm{~min}$ )

Fit peak position ( gain)

Correction for air pressure
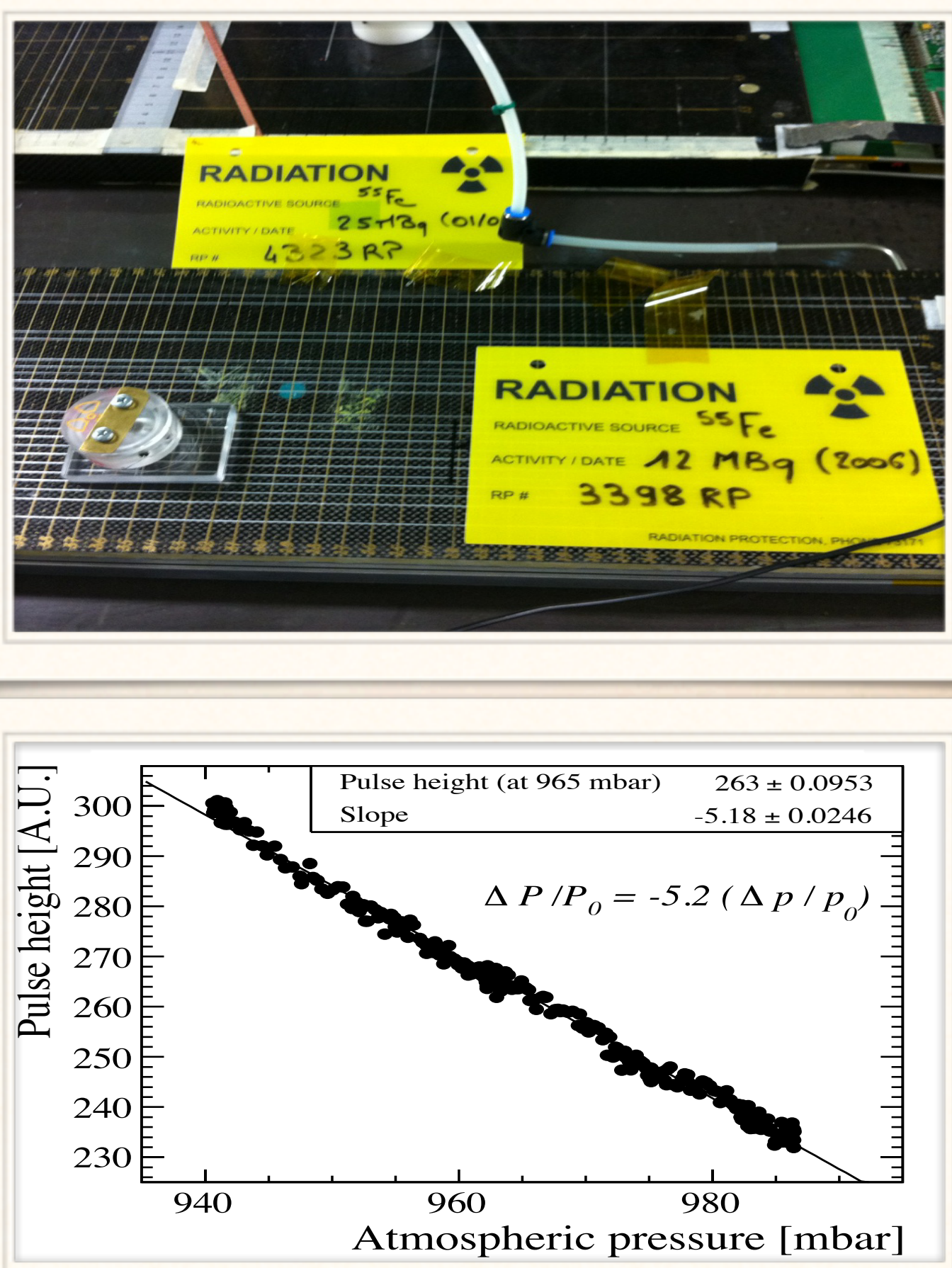


\section{GAS GAIN TRENDS}

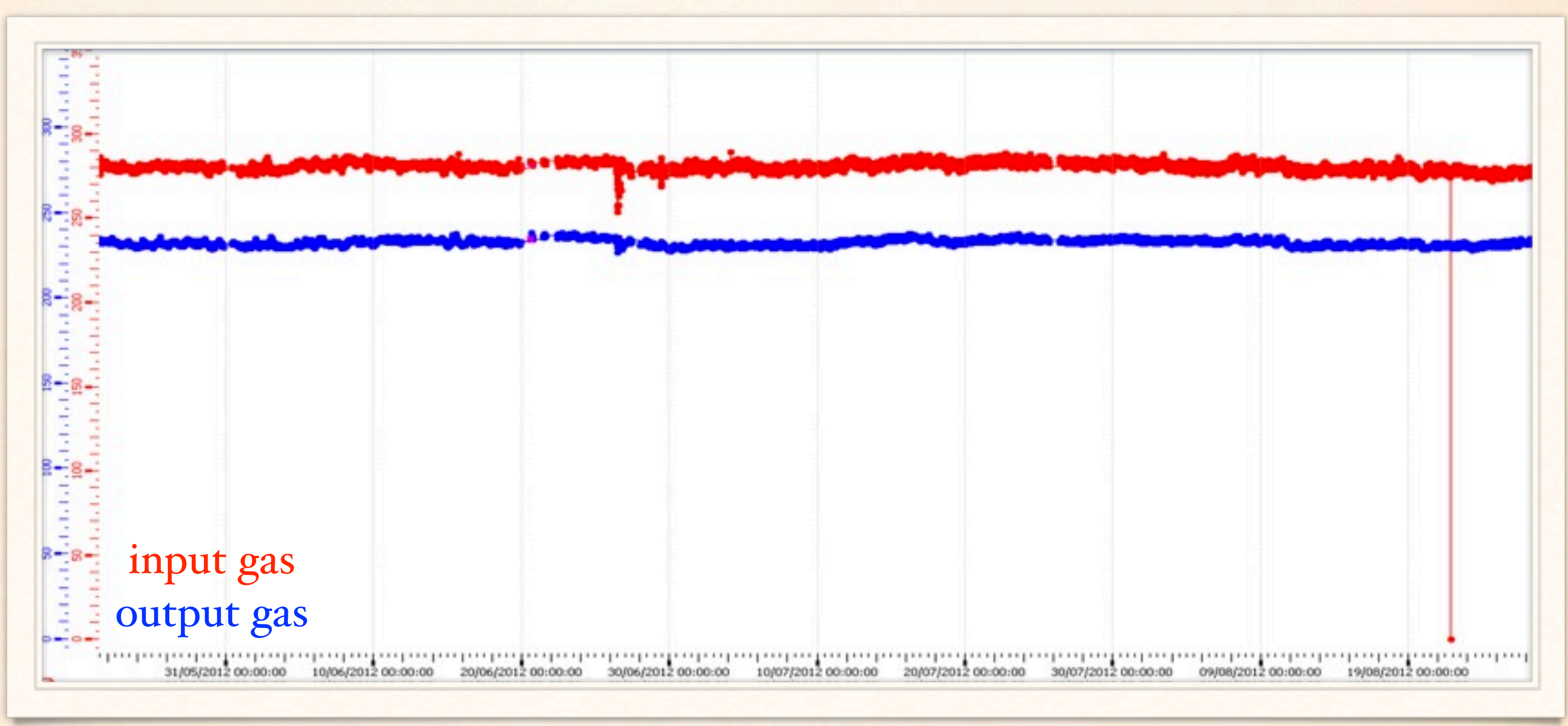

Data for $\sim 3$ months from the last year's running period

Gas gain stability is within $\pm 2 \%$

All the spikes are due to known interventions 
PERFORMANCE 


\section{ALIGNMENT}

$\hat{*}$ The design and construction of the OT detector guarantees a mechanical stability of $100(500) \mu m$ in the $x(z)$ direction.

- An optical survey measured the position of all modules after installation to $200 \mu \mathrm{m}$

An optical alignment system measures relative displacements

-8. detailed pattern mounted on the 4 corners of the C-frames

-8 the position of the C-frames is unchanged after opening and closing within $\pm 10 \mu \mathrm{m}$, and unchanged within $\pm 20 \mu \mathrm{m}$ for data taking periods with opposite polarity of the $\mathrm{LHCb}$ dipole magnet

- Software alignment with reconstructed tracks (see the talk of M. Martinelli on 'Tracking and Alignment of the LHCb detector') 


\section{5-50-75 BUNCH CROSSING}

*. Three consecutive intervals of 25 ns are readout upon a positive L0-trigger.

*. Only the first hit in the readout window is recorded

Distributions obtained in the 75 ns bunchcrossing period are close to those observed with only one single bunch crossing: free of spillover

- Double pulse:

-8 in 30 to $40 \%$ of all hits

.\& 30 ns later

-8 due to: multiple ionizations, reflections, photon feedback
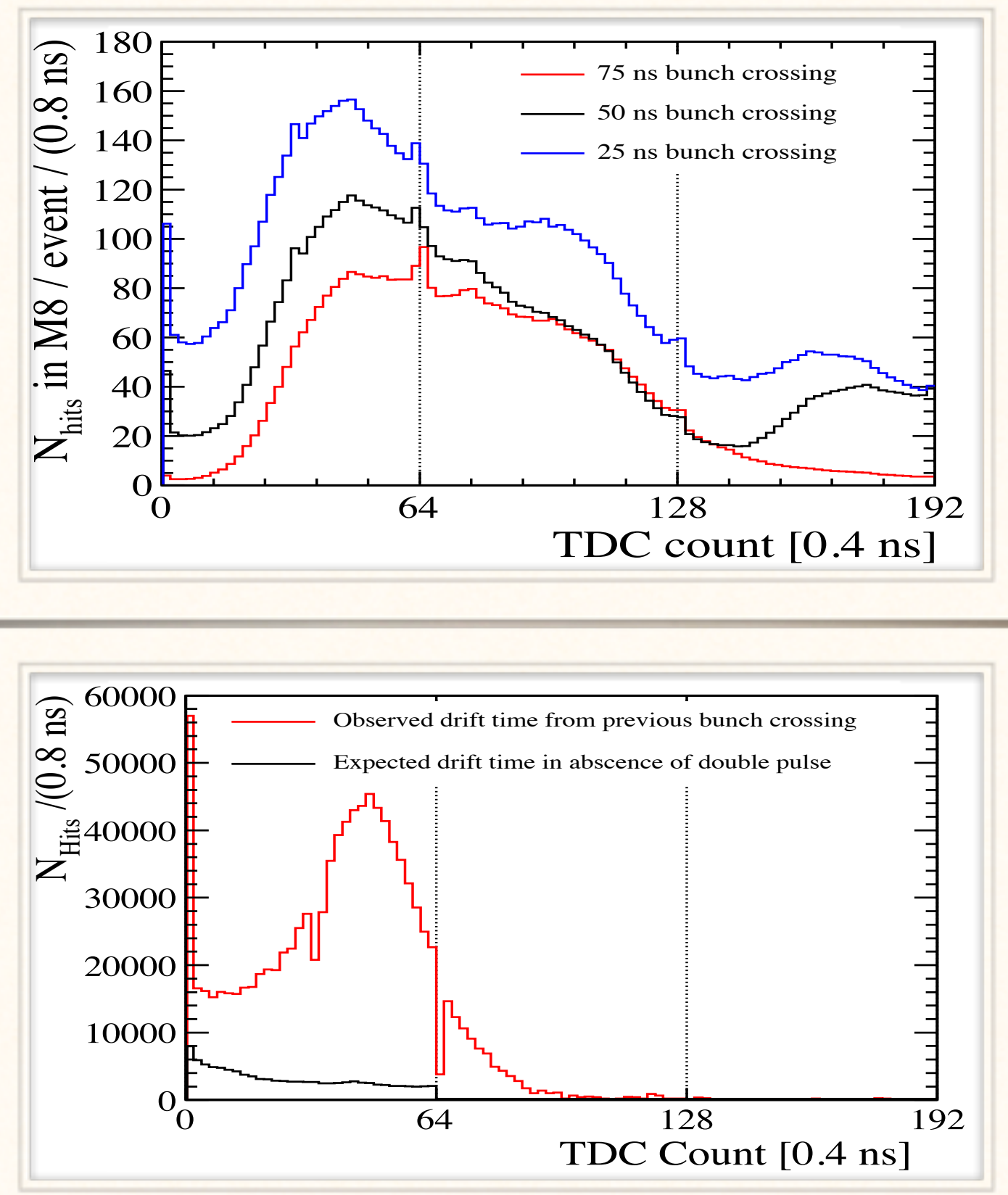

Drift time spectrum understood, and occupancy under control 


\section{OT OCCUPANCY}

. In absence of spill over: 3 to 15\% occupancy (at 50 ns)

. $30 \%$ of all hits are due to spillover

Most of the hits originate from secondary charged particles produced in interactions with material

Hits from tracks that originate from the genuine pp interaction or a subsequent particle decay are predominantly located close to the interaction region (T1 / T2 / T3 : 27.7\% / 27.1\% / 25.7\%)

Despite the high occupancy: excellent tracking efficiency
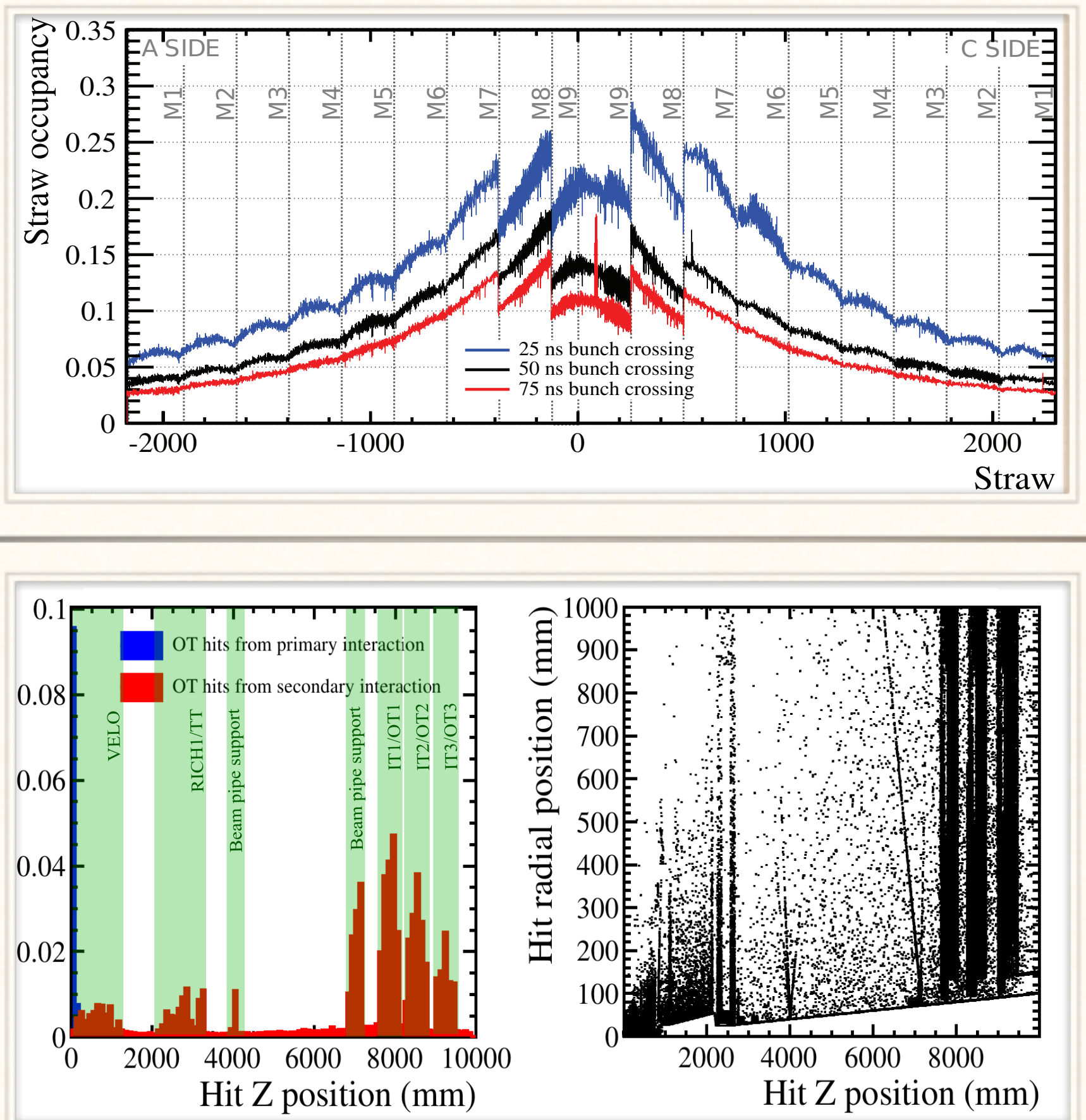


\section{SINGLE HIT EFFICIENCIES}

A high single-hit efficiency is crucial, as it affects the tracking efficiency

. Efficiency is defined as the number of observed hits in a particular detector region over the number of expected hits in the same region

* Limited probability to ionise the gas at the straw tube edges

Average efficiency for single hits at the center of the straw for a given module : $99.2 \%$

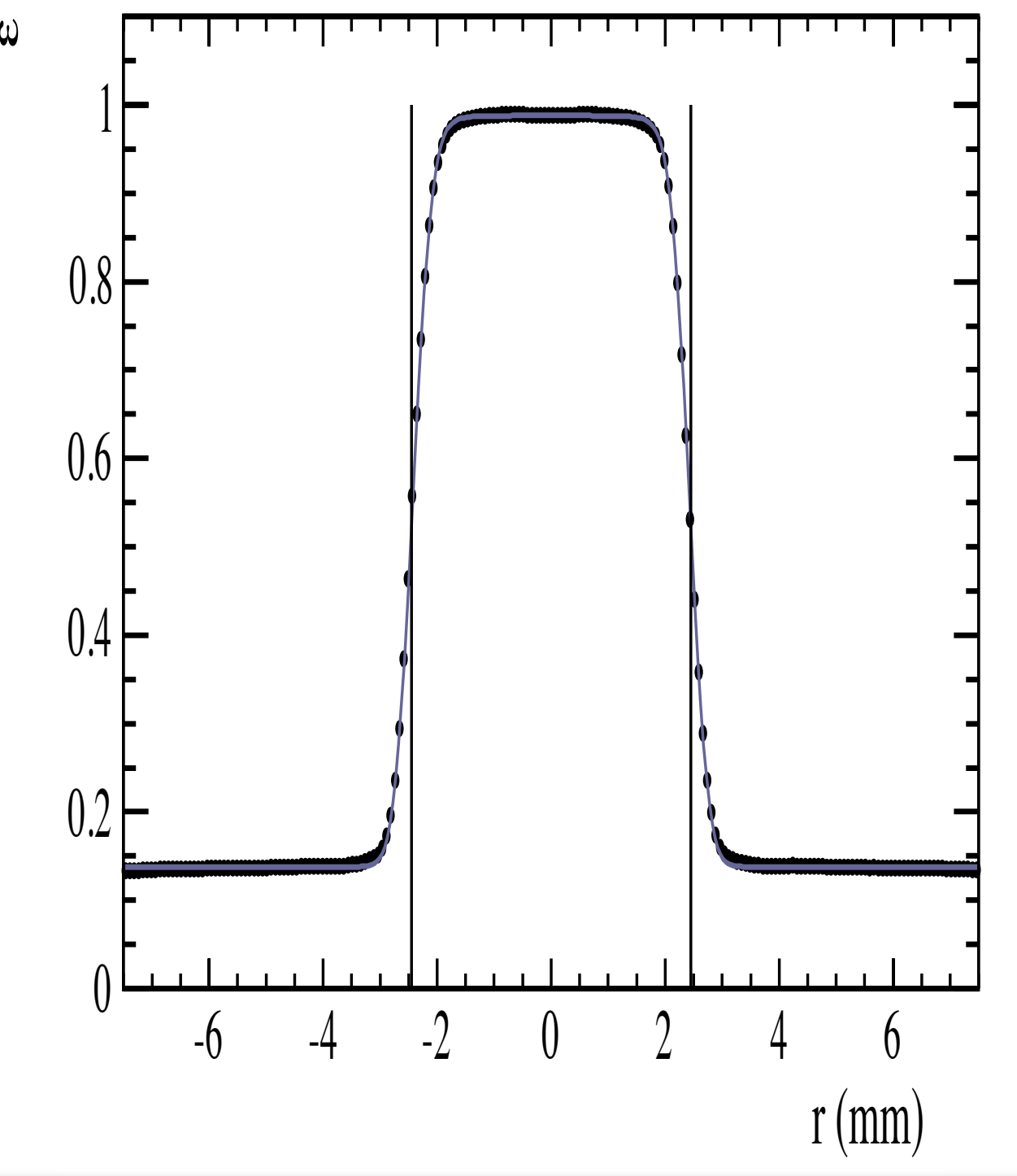




\section{OT HIT RESOLUTION}

- For a given track, the drift-time and the hit position in a straw are predicted, and compared with the measured drift-time and position

* Drift-time residual distribution width: 3ns (dominated by the ionization and drift properties in the counting gas)

- Hit distance residual distribution width: 210 $\mu \mathrm{m}$ (close to the design value of $200 \mu \mathrm{m}$ )

. Potential improvement: two monolayers within one detector module are allowed to be relatively displaced to each other in the alignment procedure
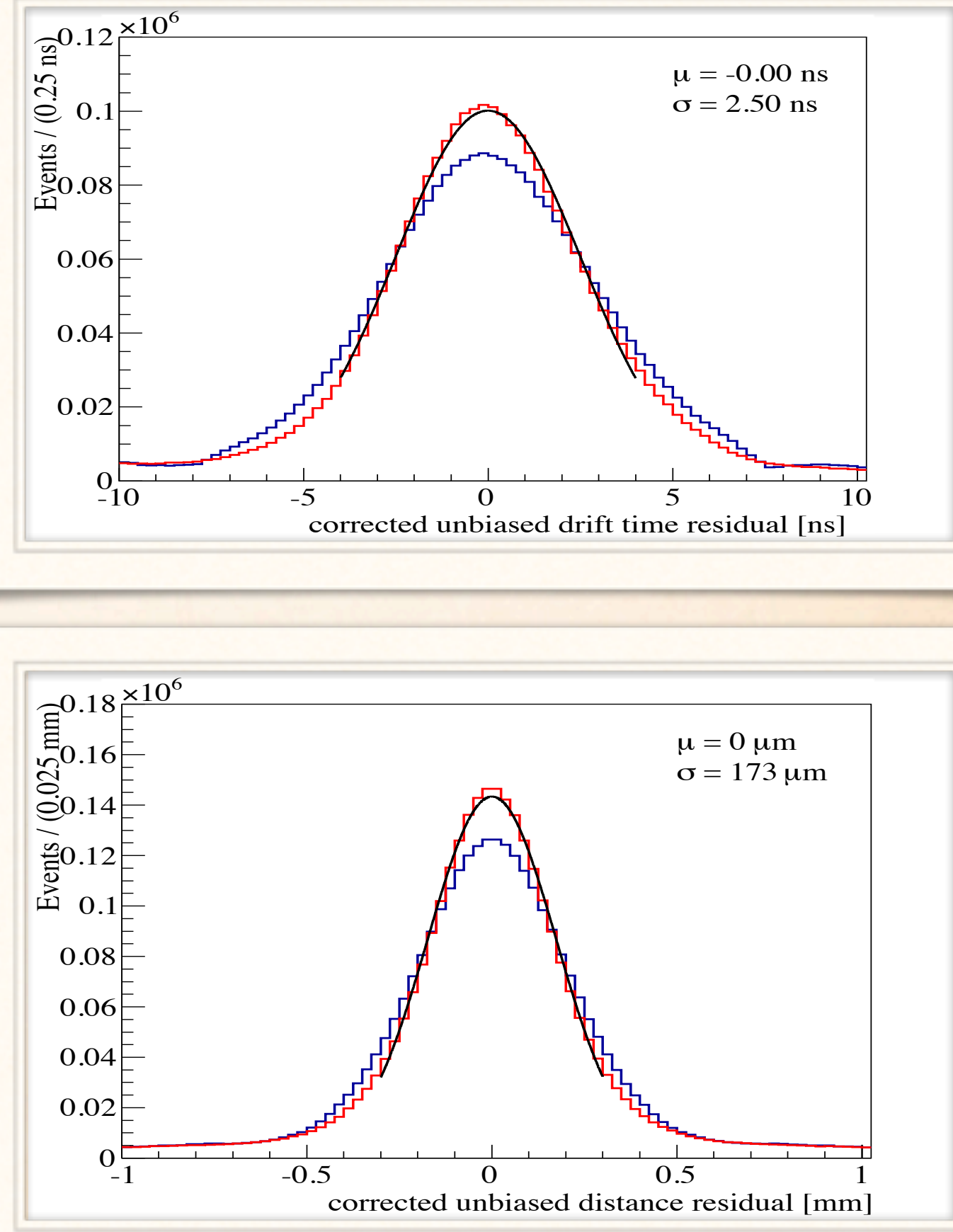


\section{RADIATION HARDNESS}




\section{AGEING EFFECTS}

${ }^{90} \mathrm{Sr}$ Source

Irradiation of modules in the lab (before LHCb): ageing visible

Radiation damage:

•r. Half moon shape

.\&. The gain loss occurs mainly upstream the source position

-6. Ozone created in avalanches protects the wires downstream

. Bachmann, et al., Nucl.Instrum.Meth. A 617 (2010) 202; N. Tuning, et al., Nucl.Instrum.Meth. A656 (2011) 45-50S.

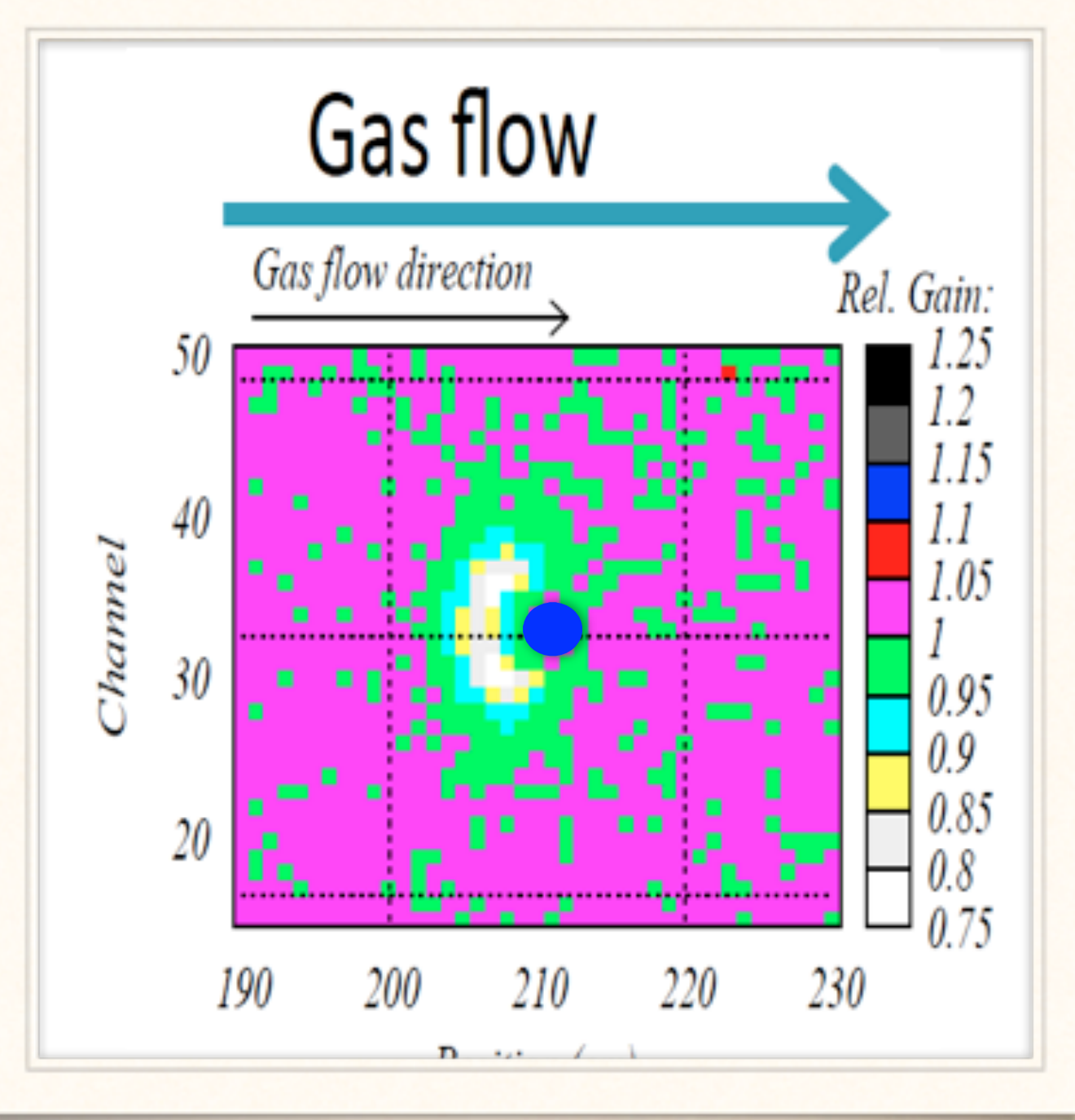

Ageing seen in lab: due to outgassing of the plastifier of the Araldite AY103-1glue 


\section{AGEING TESTS IN SITU}

(1) 2 methods to determine signal loss:

Measure response to ${ }^{90} \mathrm{Sr}$ source

·f only a few modules at a time

-6 only at shutdown periods

.8 from hours to weeks

Measure efficiency point with variating ASDBLR threshold for each of the 12 module layers individually with tracking.

• entire OT

•. during nominal LHC operation

•f $\quad 2$ hours for scanning all layers

. D. van Ejik et al., Nucl.Instrum.Meth. A685 (2012) 62-69 


\section{SCANNING FRAME}

Using two ${ }^{90} \mathrm{Sr}$ source to measure gain

* Measure response to radioactive source (current profile) for each straw channel for each position along $Y$

* Correct for air pressure

* No beam induced ageing visible so far

- Test at each technical stop of the LHC with irradiation source

- Short irradiation tests during technical stop

(.) No sign of ageing: gain variation uniform

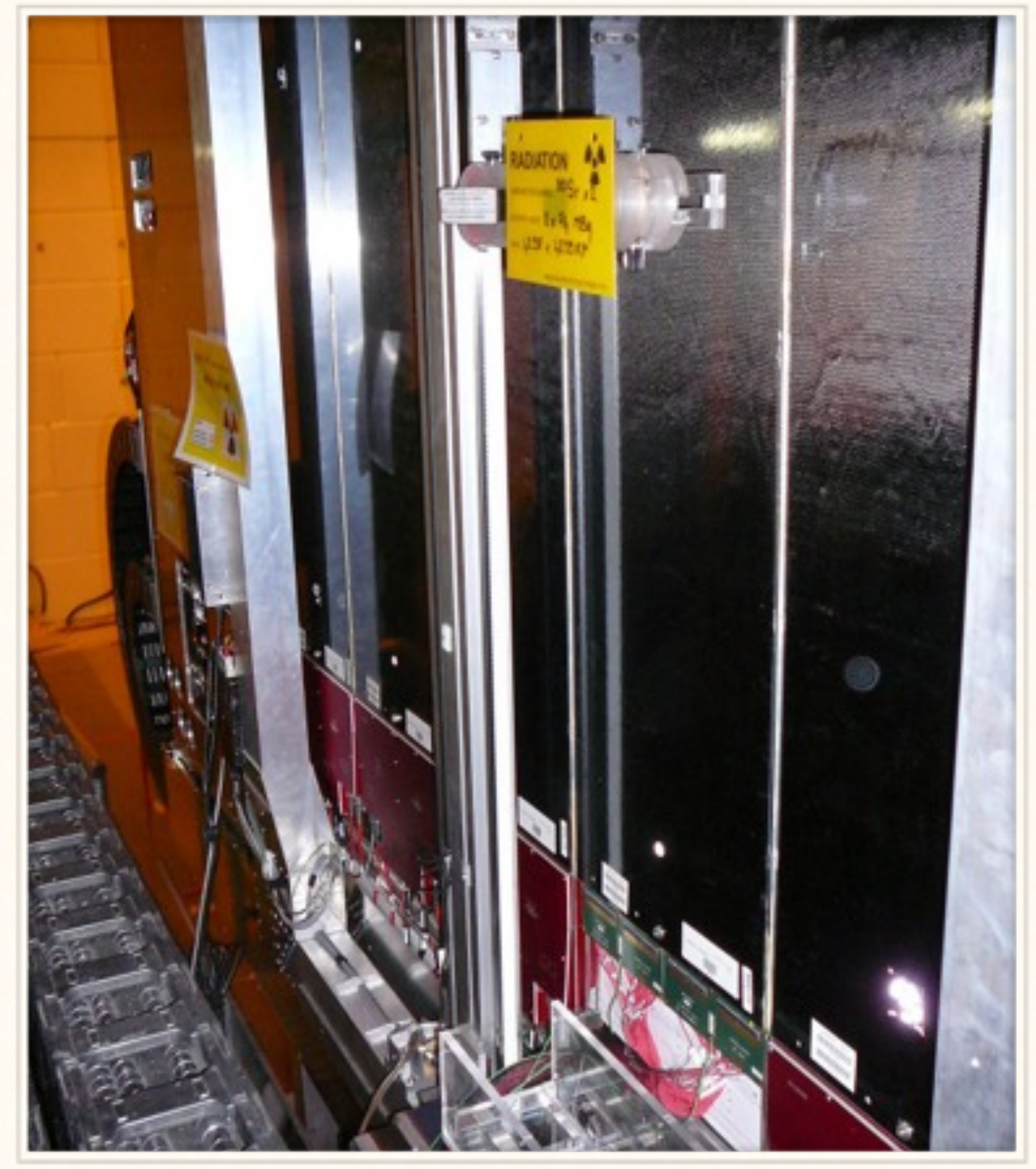
within $\pm 5 \%$ 


\section{NO AGEING IN 4 MODULES}

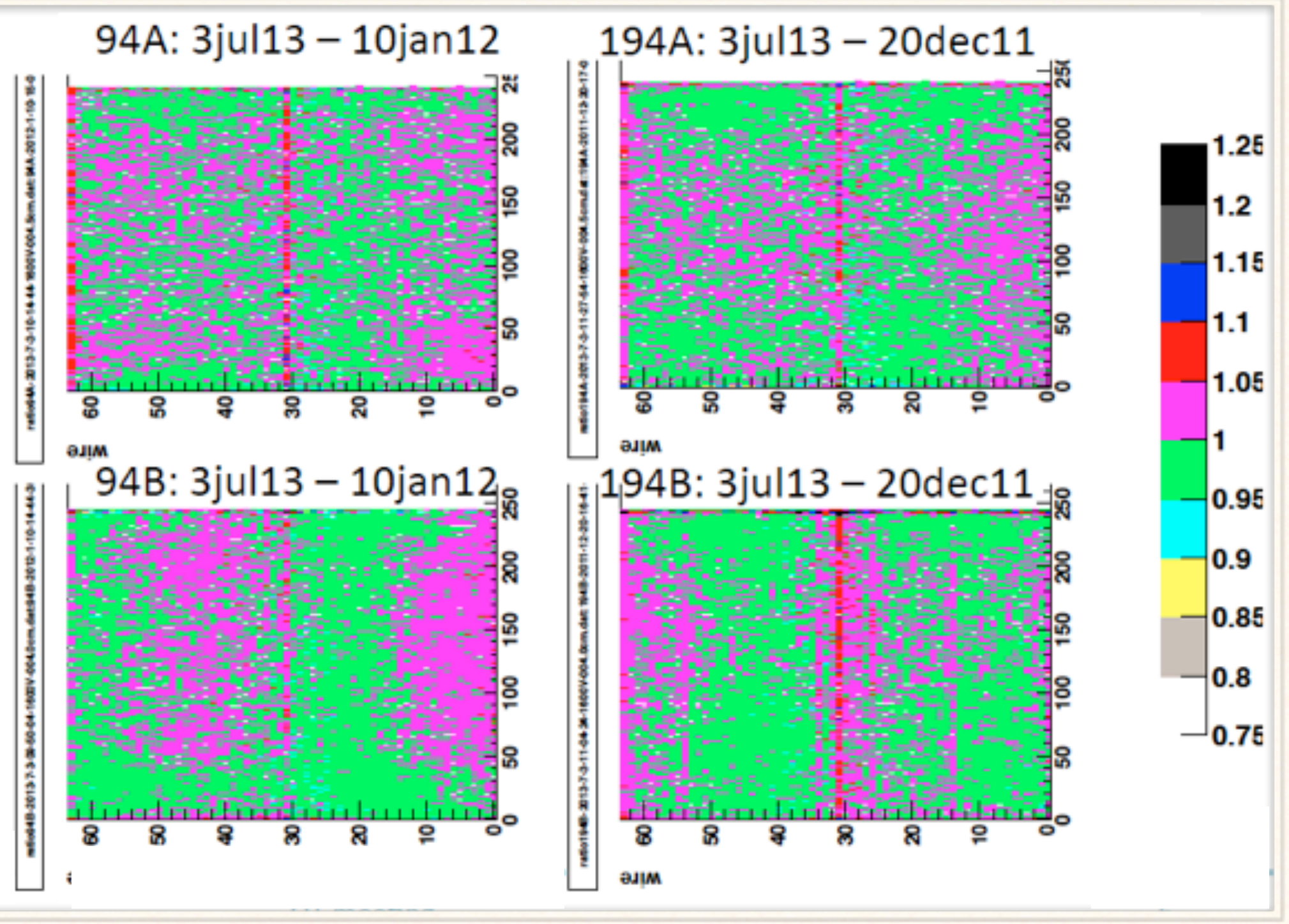

In $2012, \mathrm{LHCb}$ received $-2.2 \mathrm{fb}^{-1}$ of data and the hottest region received about $0.08 \mathrm{C} / \mathrm{cm}$

( and $3.5 \mathrm{fb}^{-1}$ of data;

$0.12 \mathrm{C} / \mathrm{cm}$ in 2OII+2OI2)

No ageing 


\section{THRESHOLD LAYER SCAN}

(3) Measure hit efficiency with tracks by scanning threshold values per layer.

(.) Determine with S-curve for each $12 \times 12 \mathrm{~cm}^{2}$ bin half-efficiency point (HEP)

(. Convert HEP to relative gain for each bin

Correct relative gain by air pressure

* Take gain ratio for each OT bin

Q Get ratio map for each layer

(. So far no beam induced radiation damage observed for installed detector.
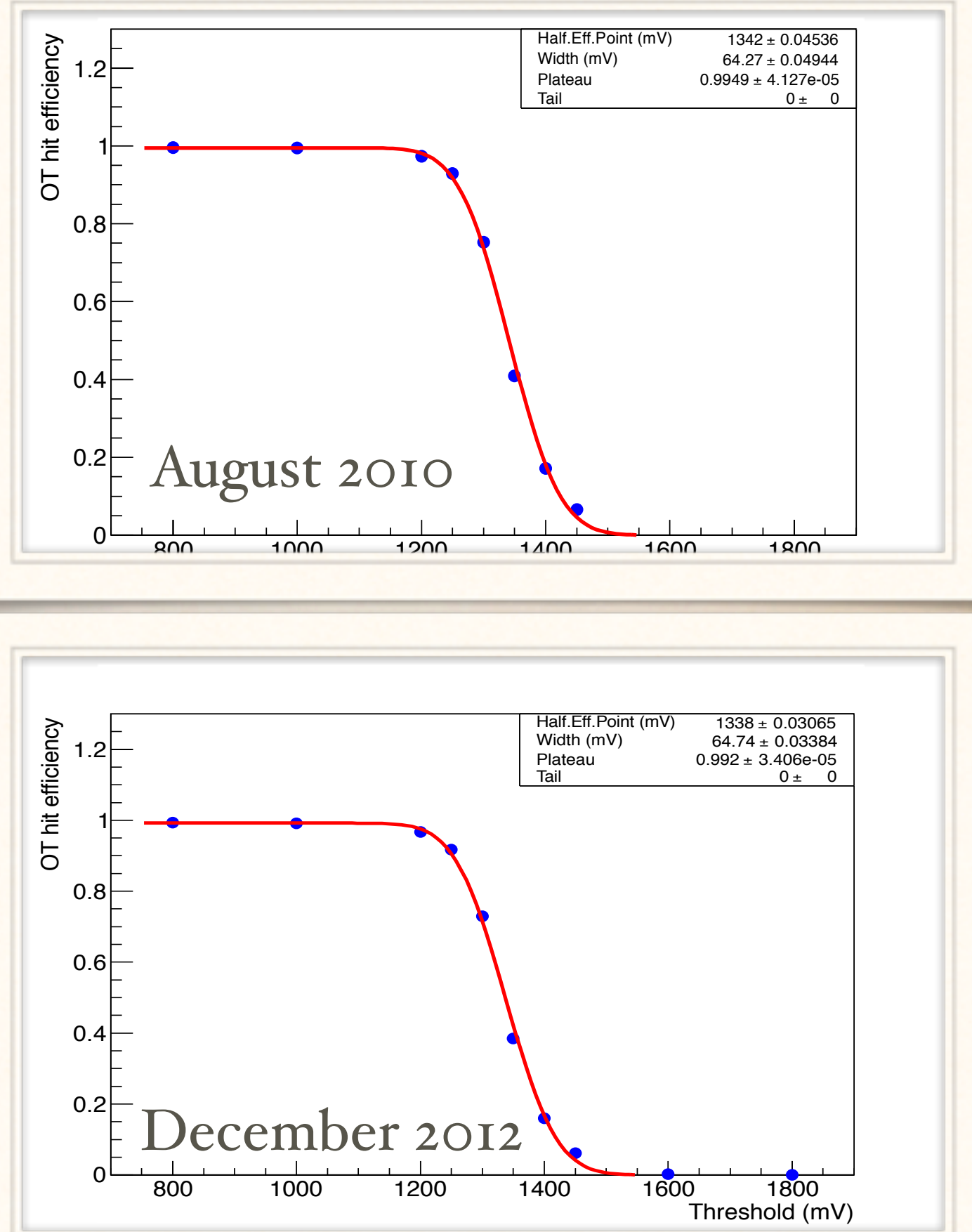


\section{SUMMARY}

Very reliable operation during the $2010,2011,2012$ LHC running periods

No significant failures of the LV, HV and gas systems.

The fraction of working readout channels was about $99.5 \%$

Excellent performance with a single-hit efficiency of about $99.2 \%$ near the center of the straw, and a single hit resolution of about $210 \mu \mathrm{m}$.

No signs of irradiation damage have been observed. 